\title{
Controlled Fluxes of Silicon Nanoparticles to a Substrate in Pulsed Radio-Frequency Argon-Silane Plasmas
}

\author{
Carlos Larriba-Andaluz ${ }^{1,2}$ and Steven L. Girshick ${ }^{1}$ \\ ${ }^{1}$ Department of Mechanical Engineering, University of Minnesota, Minneapolis, MN 55455, \\ USA \\ ${ }^{2}$ Current address: Department of Mechanical Engineering, Indiana University-Purdue University \\ Indianapolis, Indianapolis, IN, USA
}

This is the author's manuscript of the article published in final edited form as:

Larriba-Andaluz, C., \& Girshick, S. L. (2017). Controlled fluxes of silicon nanoparticles to a substrate in pulsed radio-frequency argon-silane plasmas. Plasma Chemistry and Plasma Processing, 37(1), 43-58. http://dx.doi.org/10.1007/s11090-016-9749-7 


\begin{abstract}
It has been hypothesized that high-energy impact of very small silicon nanoparticles on a substrate may lead to epitaxial growth of silicon films at low substrate temperature. A possible means for producing such energetic nanoparticle fluxes involves pulsing an RF silane-containing plasma, and applying a positive DC bias to the substrate during the afterglow phase of each pulse so as to collect the negatively charged particles generated during the RF power on phase. We here report numerical modeling to provide a preliminary assessment of the feasibility of this scheme. The system modeled is a parallel-plate capacitively-coupled RF argon-silane plasma at pressures around 100 mTorr. Simulation results indicate that it is possible to achieve a periodic steady state in which each pulse delivers a controlled flux of nanoparticles to the biased substrate, that average particle sizes can be kept below 2-3 nm, that impact energies of the negativelycharged nanoparticles that are attracted by the applied bias can be maintained in the $\sim 1 \mathrm{eV} / \mathrm{atom}$ range thought to be conducive to epitaxial growth without causing film damage, and that the volume fraction of neutral nanoparticles that deposit by low-velocity diffusion can be kept well below $1 \%$. The effects of several operating parameters are explored, including RF voltage, pressure, the value of the applied DC bias, and RF power on and off time during each pulse.
\end{abstract}

\title{
1. Introduction
}

Recently it was reported that epitaxial growth of silicon thin films on (100)-oriented crystalline silicon substrates had been achieved by plasma-enhanced chemical vapor deposition at the unusually low substrate temperature of $175^{\circ} \mathrm{C}[1]$. The authors hypothesized that the key to achieving this result was that the film had grown not by chemical vapor deposition but rather by deposition of silicon nanoparticles that formed in the plasma.

Low-temperature plasmas consisting of a silicon precursor (typically silane) diluted in a background gas (typically a noble gas or hydrogen) are a well-known medium for synthesis of silicon nanoparticles [2-6], so it is plausible that silicon nanoparticles are formed in the radio frequency (RF) hydrogen-silane plasma employed in [1]. It has also been argued that charged nanoclusters and nanoparticles are commonly the building blocks for thin film growth in plasmaenhanced chemical vapor deposition [7].

What is less obvious is the mechanism by which these particles could deposit to grow an epitaxial film. One possible explanation is that positively charged nanoparticles, attracted by the 
sheath potential drop above the substrate, could impact with enough kinetic energy to melt the particles, so that the silicon atoms comprising the particles would then have sufficient mobility to foster epitaxial growth on an oriented crystalline substrate [8]. As particles of all sizes would experience the same sheath potential drop, this explanation would be particularly promising for very small particles, perhaps only 1-2 $\mathrm{nm}$ in diameter, for which the impact kinetic energy per atom would be much higher than for larger particles.

Indeed this possibility is suggested by molecular dynamics (MD) simulations of cluster impact on surfaces. For example, MD simulations of $\mathrm{Mo}_{1043}$ clusters ( $3 \mathrm{~nm}$ in diameter) impacting $\operatorname{Mo}(001)$ surfaces indicate a "soft touchdown" when the impact energy equals 0.1 eV/atom, a "flattening collision" at $1.0 \mathrm{eV} /$ atom, and a "meteoric impact" at $10 \mathrm{eV} / \mathrm{atom}$ [9]. In both of the higher energy cases epitaxial film growth was predicted. MD simulations of $\mathrm{Cu}_{147}$ clusters $(\sim 1.5 \mathrm{~nm}$ in diameter) impacting bare $\mathrm{Cu}(111)$ surfaces indicate that at impact velocities of $2 \mathrm{~km} / \mathrm{s}$, corresponding to $1.31 \mathrm{eV} /$ atom, the cluster melts and spreads [10]. These simulations also indicate that a cluster impacting with only thermal velocity retains its crystalline structure, while a cluster impacting at $4 \mathrm{~km} / \mathrm{s}$ (5.26 eV/atom) causes considerable substrate damage. MD simulations of 5-nm-radius $\mathrm{Si}$ nanoparticles (containing $31,075 \mathrm{Si}$ atoms) impacting $\mathrm{H}$ passivated Si substrates at velocities below $\sim 2000 \mathrm{~m} / \mathrm{s}$ (corresponding to energies below $\sim 0.5$ eV/atom) indicate that the particles retain their discrete identity but undergo an internal crystalline phase transition at impact velocities above $1250 \mathrm{~m} / \mathrm{s}(0.23 \mathrm{eV} /$ atom $)$ [11].

While one expects the details of these cluster-substrate impact interactions to depend on the materials of which the cluster and substrate are composed as well as on their initial structure and the substrate temperature, one can anticipate from these results that nanoparticles composed of the same material as an oriented crystalline substrate, and impacting the substrate with kinetic energies on the order of $1 \mathrm{eV} /$ atom, could potentially foster homoepitaxial film growth without causing film damage.

Whether or not this hypothesis is correct, one problem is that most particles in nonthermal plasmas are expected to charge negatively, because of the higher mobility of free electrons compared to ions, and under typical conditions negative particles are electrostatically trapped by the electric field in the plasma and thus are difficult to collect on the electrodes.

We here propose a possible way to avoid this problem: pulsing the plasma, and biasing the substrate positively during the afterglow phase of each pulse so as to collect the negatively 
charged particles generated during the pulse on-phase. (Equivalently, an opposing electrode could be biased negatively.) This method might have the additional advantage that by controlling the pulse on-time one could control the size attained by nanoparticles that nucleate and grow during each pulse, thereby limiting the size to the 1-2 $\mathrm{nm}$ diameter range that may be needed to achieve high impact energies per atom, while also avoiding the high impact momentum, causing substrate or film damage, that might result from impact of larger particles.

In this paper we use numerical modeling to provide a preliminary assessment of the feasibility of this scheme. We model a pulsed, capacitively-coupled parallel-plate radio frequency (RF) argon-silane plasma, at a total pressure around 100 mTorr. We explore the possibility of achieving a periodic steady state such that the flux of nanoparticles delivered to the biased substrate is reproducible from pulse to pulse, and we examine the effects of several adjustable plasma and pulse operating parameters on results including the distribution of nanoparticle impact energies (eV/atom), the fraction of deposited nanoparticles that impact by electrostatic drift as opposed to Brownian diffusion, and deposition rates.

\section{Numerical model}

We utilize a previously developed one-dimensional numerical model for a capacitivelycoupled parallel-plate RF argon-silane plasma in which silicon nanoparticles nucleate and grow. Briefly, the model, which is described in more detail in refs. [12-14], self-consistently couples a fluid plasma model with a detailed aerosol model that treats particle nucleation by chemical reactions, particle growth by coagulation and surface reactions, particle charging, and particle transport by effects including electric force, neutral drag, ion drag, Brownian diffusion, thermophoresis and gravity. The electron energy equation is solved under the assumption of a Maxwellian velocity distribution, and Poisson's equation for the electric field is solved over the onedimensional space between two infinite parallel-plate electrodes. The model solves population balance equations for electrons, argon ions and metastables, hydrogen species, and silicon hydrides (neutral and ionic) containing one or two Si atoms, with the nucleation rate equated to the rate of formation of anions containing at least three Si atoms. Clusters containing at least three $\mathrm{Si}$ atoms, corresponding to a diameter of about $0.5 \mathrm{~nm}$, are treated as "nanoparticles," for which the aerosol general dynamic equation is solved using a sectional method [14]. We assume that once these nanoparticles form they do not dissociate. A simple model for particle surface growth 
is assumed, based on a sticking coefficient formalism. Particle charging by electron and ion attachment is modeled assuming the orbital motion limited theory [15], with the single-particle charge assumed to be limited according to Gallagher's model for Si particle charging [16,17]. Other charging mechanisms, such as UV photodetachment and secondary electron emission, are neglected. Coagulation is modeled accounting for the sizes and charge states of the colliding nanoparticles, and considering the effect of image potentials on coagulation between charged and neutral nanoparticles $[18,19]$.

In previous work this model was used to conduct numerical simulations of the spatiotemporal evolution of a continuously running RF argon-silane plasma [14]. Here we modify the model to treat pulsing of the RF plasma, with biasing of one of the parallel-plate electrodes during the afterglow phase of each pulse, as illustrated schematically in Fig. 1. To avoid numerical difficulties during the brief period following turn-off of the RF voltage, when free electrons rapidly escape the system by diffusion, we do not instantaneously apply the DC bias at the beginning of the RF-off phase, but instead delay the introduction of the bias by a small time, allowing effectively all of the electrons to be lost. For the conditions of these simulations this delay time

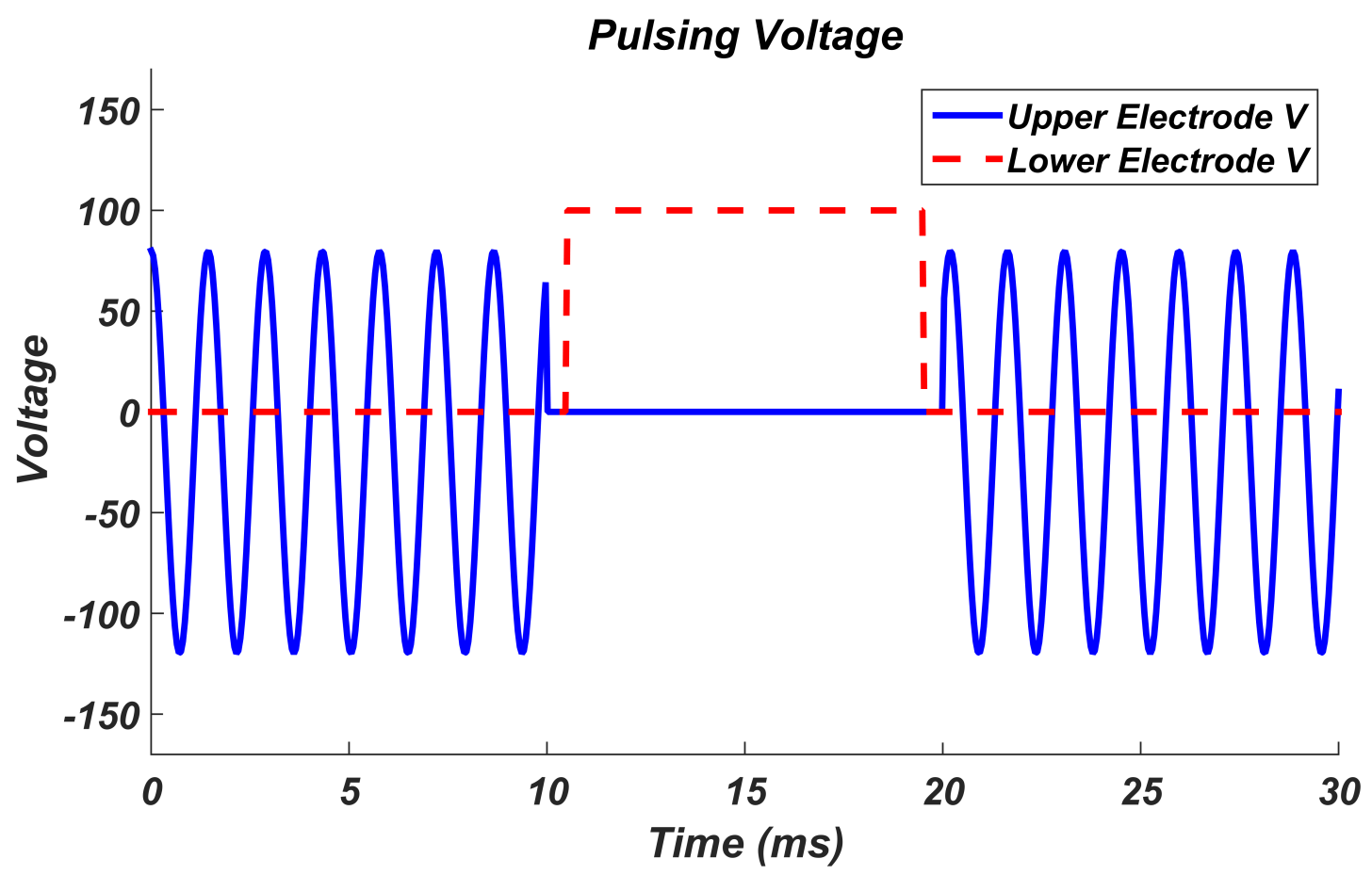

Figure 1. Illustration of pulsing scheme. Positive DC bias is applied to the lower electrode when $\mathrm{RF}$ power is off. Bias is removed when RF power is on. Asymmetry of RF voltage is due to DC self-bias on upper electrode that is self-consistently calculated. 
was less than $0.1 \mathrm{~ms}$ in all cases.

We consider a base case for which the plasma is at $13.56 \mathrm{MHz}$ frequency, with $100 \mathrm{~V}$ (amplitude) RF power applied across a 4-cm electrode gap, a pressure of 100 mTorr, an argonto-silane ratio of 30:1 with a flow velocity at the top (showerhead) electrode of $27 \mathrm{~cm} / \mathrm{s}$, a gas (heavy species) temperature of $300 \mathrm{~K}$, a DC bias of $+100 \mathrm{~V}$ applied to the film substrate (lower electrode) during the RF-off phase of each cycle, and a pulse frequency of $100 \mathrm{~Hz}$ with a duty cycle of $50 \%$.

\section{Results and discussion}

In each simulation, the plasma is initially free of nanoparticles. Under many sets of conditions we find that after an initial transient period the plasma-nanoparticle system reaches a periodic steady state in which the flux of nanoparticles delivered to the substrate is reproducible from pulse to pulse. We first show results for the base case during a single pulse after the system has reached such a periodic steady state, then consider the approach to steady state, and finally discuss the effects of operating parameters on system performance.

\subsection{System evolution during a single pulse}

For a single pulse which occurs after the system has reached periodic steady state under base case conditions, Figs. 2-5 show the temporal evolution of spatial profiles across the electrode gap of key properties of the plasma-nanoparticle system. Figure 2 shows results at the end of the 5-ms phase during which the RF power is on; Fig. 3 shows corresponding results $0.2 \mathrm{~ms}$ into the afterglow phase, after the RF power has been turned off and the DC bias turned on; Fig. 4 shows results $0.4 \mathrm{~ms}$ into the afterglow; and Fig. 5 shows results late $(4.8 \mathrm{~ms})$ in the afterglow. In each of these figures, graph (a) shows density profiles of negative, neutral, and positivelycharged nanoparticles; graph (b) shows profiles of the particle size distribution function $d N / d\left(d_{p}\right)$, where $N$ is particle density and $d_{p}$ particle diameter, and average charge; and graph (c) shows density profiles of all charge carriers, including electrons, positive ions (the sum of all cations), negative ions (the sum of all anions), total charge carried by negatively-charged nanoparticles, and total charge carried by positively-charged nanoparticles.

As seen in Fig. 2(a), when the RF power is on, negatively-charged nanoparticles are trapped in the center of the plasma by the electric field, while neutral nanoparticles follow a den- 

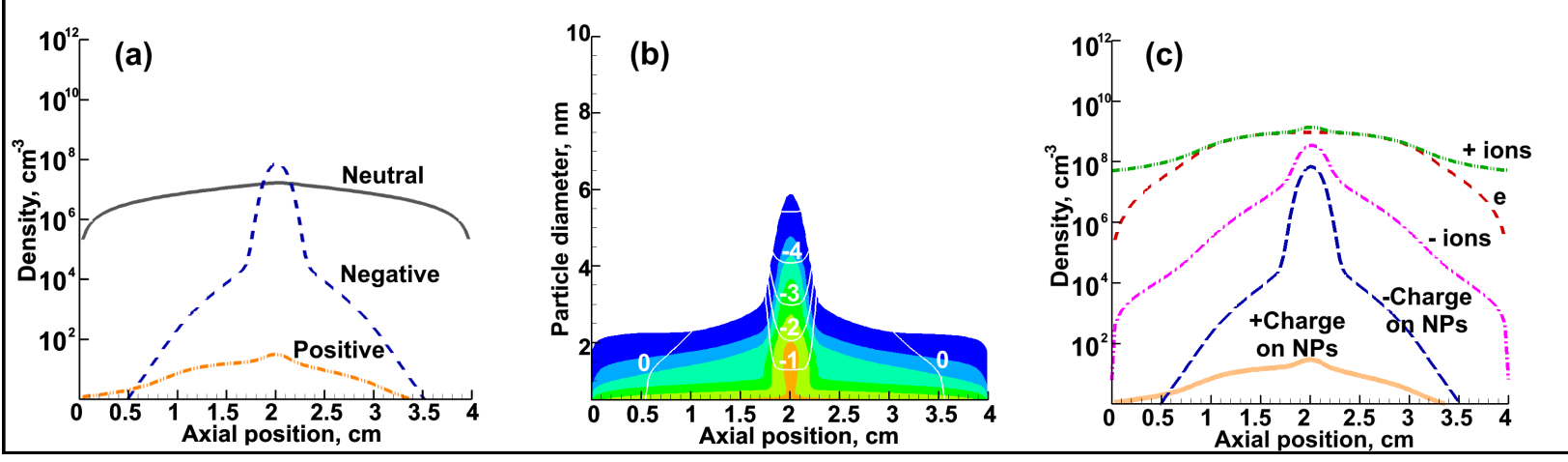

Figure 2. Profiles across the electrode gap at the end of RF power on phase of a cycle, base case: (a) total densities of neutral, negative and positive nanoparticles; (b) particle size distribution (color contours) and average charge (white lines); (c) charge carriers.

sity profile driven by Brownian diffusion. Since positive nanoparticles are created by ion attachment to neutral nanoparticles, the peaks in their density profiles are closely related, even though the positive nanoparticles are repelled from the center by the electric field. Fig. 2(b) shows that when particles grow beyond 1 or $2 \mathrm{~nm}$ in diameter the vast majority are negatively charged and trapped in the center of the plasma. As seen in Fig. 2(c), the density profiles of all charged species peak in the center, satisfying quasi-neutrality via solution of Poisson's equation. The density profiles of ions are broader than those of nanoparticles of the same polarity, because the nanoparticles, being more massive than ions, are less mobile.

When the RF power is switched off and positive DC bias is applied to the lower electrode, the negative nanoparticles, instead of being trapped in the center, are now attracted toward the biased electrode. Figure 3 shows density profiles for the same set of properties as in Fig. 2, but now $0.2 \mathrm{~ms}$ into the afterglow phase of the same pulse. At this time, as seen in Fig. 3(a), the negative nanoparticles are pulled by the positive DC bias toward the lower electrode. Their peak density is now located slightly below the midplane, and they have established a flux to the lower electrode. In contrast the profile of neutral nanoparticles in Fig. 3(a) is barely changed from Fig. 2(a), as the neutral nanoparticles are not (directly) affected by the change in the electric field. The neutral nanoparticle density decays with time due to loss by diffusion to both electrodes, but on a much longer time scale than the rapid transport of negative nanoparticles by the electric field. Meanwhile, the positive nanoparticles, though much less abundant than negative or neutral nanoparticles, exhibit an interesting behavior: their density profile in Fig. 3(a) indicates that they flow toward the lower electrode, in spite of the positive DC bias applied to that electrode. The reason for this behavior is that the density profile of positive nanoparticles is coupled to that of 

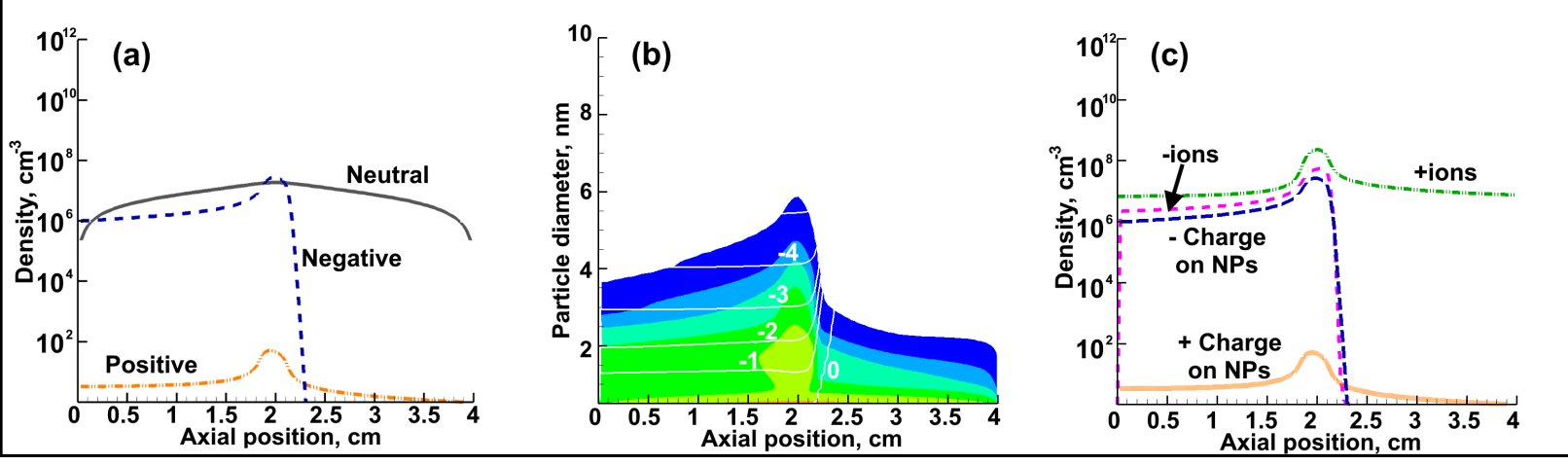

Figure 3. Profiles across the electrode gap, $0.2 \mathrm{~ms}$ into the afterglow phase of a pulse, base case: (a) total densities of neutral, negative and positive nanoparticles; (b) particle size distribution (color contours) and average charge (white lines); (c) charge carriers.

negative nanoparticles, via Poisson's equation. The fact that both negative and positive nanoparticles flow toward the lower electrode thus represents a kind of ambipolar diffusion.

In Fig. 3(b) one sees that the nanoparticles arriving at the lower electrode are on average larger than those "left behind" in the upper half of the electrode gap. This is a consequence of the fact that larger nanoparticles are much more likely than smaller ones to be negatively charged, and it is the negative nanoparticles that are attracted by the positively biased electrode, while neutrals diffuse to both electrodes. One also observes that, within the population of negative nanoparticles that flow toward the lower electrode, larger nanoparticles are transported more slowly than smaller ones, caused by the inverse relationship between mobility and particle size. Thus the size distribution profile in the lower half of the electode gap exhibits a classification by particle size.

Figure 3(c), which shows density profiles of all charge carriers, indicates that by $0.2 \mathrm{~ms}$ into the afterglow virtually no free electrons remain in the system. Numerical simulations of a plasma afterglow under similar conditions, reported in [13] and in more detail in [20], show that in the early afterglow electrons and ions diffuse together by ambipolar diffusion, but then, after $30-50 \mu \mathrm{s}$, undergo a transition to free diffusion in which the electrons diffuse much more rapidly than the ions. Meanwhile, electron temperature relaxation occurs very rapidly, decaying to the background gas temperature on a time scale of 1-3 $\mu \mathrm{s}$. Numerical simulations by Ramamurthi and Economou of the afterglow of a pulsed pristine RF argon plasma at even lower pressure (20 mTorr) show similarly rapid electron energy relaxation, due primarily to inelastic collisions of electrons with Ar and Ar* metastables [21], and also similar results for the drop in electron density, and these results for both electron density and temperature are in reasonable agreement with 
experimental studies of pulsed RF plasmas [22]. The rapid drop in electron temperature causes a sharp reduction in electron attachment to nanoparticles. For negative nanoparticles, where electron attachment requires overcoming the negative particle potential, the time scale for attachment increases from $\sim 1 \mu \mathrm{s}$ for an electron temperature of $1 \mathrm{eV}$ to $\sim 1 \mathrm{~s}$ for an electron temperature of $0.025 \mathrm{eV}$ [20]. For neutral nanoparticles a reduction in attachment rate also occurs but is less severe, as it scales on the electron thermal velocity. However the smaller average size of neutral nanoparticles in these simulations compared to negative ones implies a smaller attachment crosssection. Taking all these effects together, one can conclude that the rapid loss of electrons from the system occurs mainly by diffusion, with some additional contribution due to attachment to neutral and positive nanoparticles.

Thus in this early afterglow phase, before all of the negative nanoparticles have been collected, the charge carriers consist only of ions and nanoparticles, both negative and positive. Comparing Figs. 3(c) and 2(c), one notes decay in the positive ion density, since ionization by electron impact has ceased and positive ions are lost both by diffusion to the electrodes and by attachment to nanoparticles. However, this decay is much slower than is the loss of electrons. Meanwhile, the flow of negative ions and nanoparticles to the lower electrode creates the ambipolar diffusion noted above, together with a strong positive space charge in the upper part of the electrode gap, from which all of the negative species have been removed.

Figure 4 shows the corresponding results $0.4 \mathrm{~ms}$ into the afterglow. All of the trends noted with respect to Fig. 3 continue as the negative nanoparticles are collected by the lower electrode. In Fig. 4(a) one sees that the density profile of negative nanoparticles has shifted notably
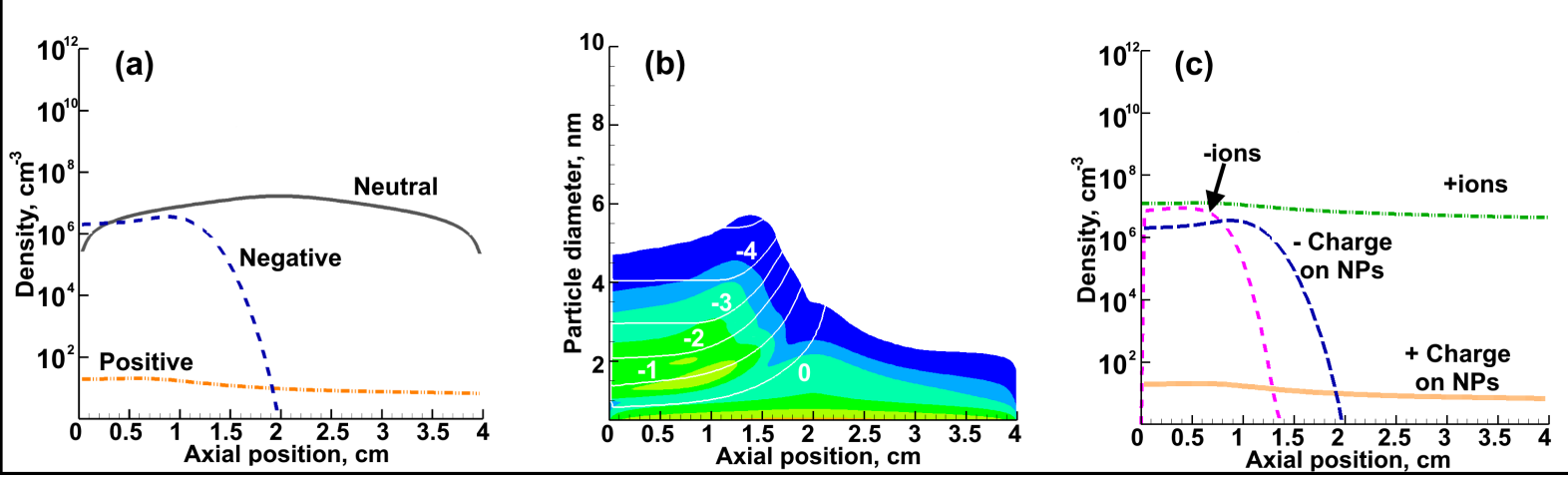

Figure 4. Profiles across the electrode gap, $0.4 \mathrm{~ms}$ into the afterglow phase of the same pulse as in Figs. 2-3: (a) total densities of neutral, negative and positive nanoparticles; (b) particle size distribution (color contours) and average charge (white lines); (c) charge carriers. 
toward the lower electrode compared to Fig. 3(a); the density profile of neutral nanoparticles is barely changed; and the positive nanoparticle density profile continues to exhibit a coupling with that of the negative nanoparticles. One can observe a small increase in the total number of positive nanoparticles, which can be attributed to ion attachment to neutral nanoparticles. In Fig. 4(c) it is seen that the anions are collected more rapidly than the negative nanoparticles, because of their higher mobility.

Finally all of the negative nanoparticles have been collected. Figure 5 shows the density profiles $4.8 \mathrm{~ms}$ into the afterglow. The remaining nanoparticles are all neutral, and the only remaining charge carriers are positive ions. The reason that the positive nanoparticles are lost from the system before the positive ions, in spite of the higher mobility of the ions, is simply that the initial positive ion density at the start of the afterglow phase is much higher than that of the positive nanoparticles. The density profile of positive ions in Fig. 5(c) now has a positive slope due to the applied field, although this is difficult to see on the logarithmic scale of the figure.

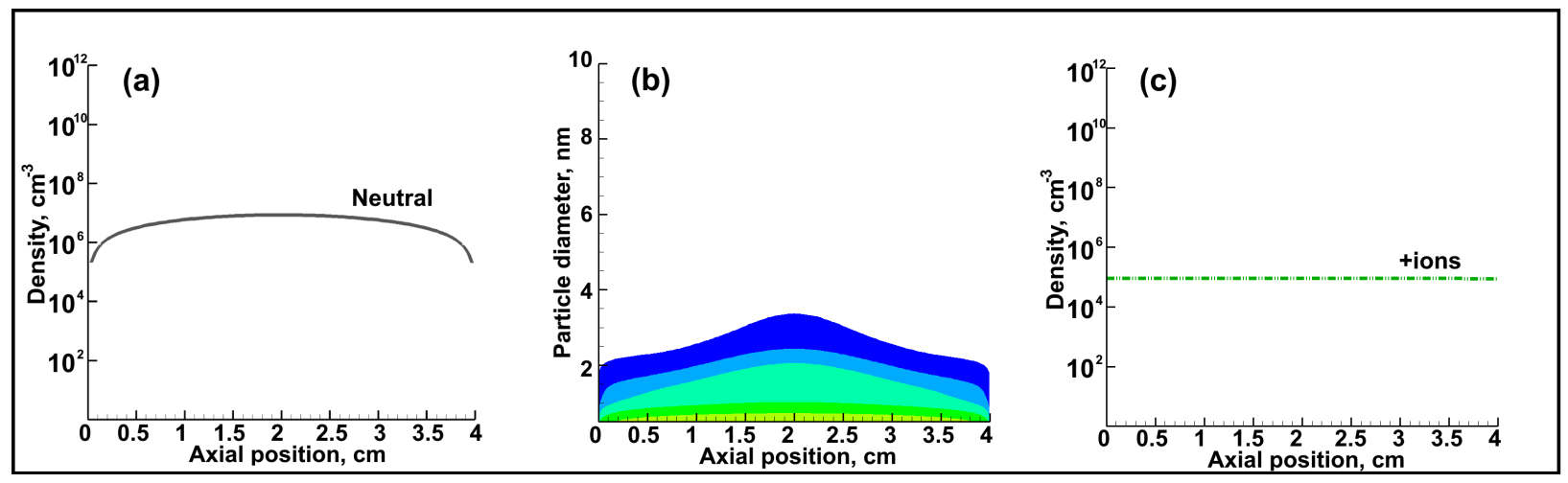

Figure 5. Profiles across the electrode gap, $4.8 \mathrm{~ms}$ into the afterglow phase of the same pulse as in Figs. 2-4: (a) total density of neutral nanoparticles (only neutral nanoparticles remain); (b) particle size distribution; (c) charge carriers.

\subsection{Approach to periodic steady state}

The results presented in the previous section are all for a single RF power on and off cycle that occurs after the system has reached a periodic state. We find that under a broad range of conditions such a periodic state is rapidly achieved. One way to demonstrate this is by examining the time-varying volume fluxes of nanoparticles to the lower electrode, as shown for base case conditions in Fig. 6. At time zero the argon-silane gas mixture is flowing and the RF power is turned on. Nanoparticles begin to form, and neutral nanoparticles diffuse to the lower elec- 


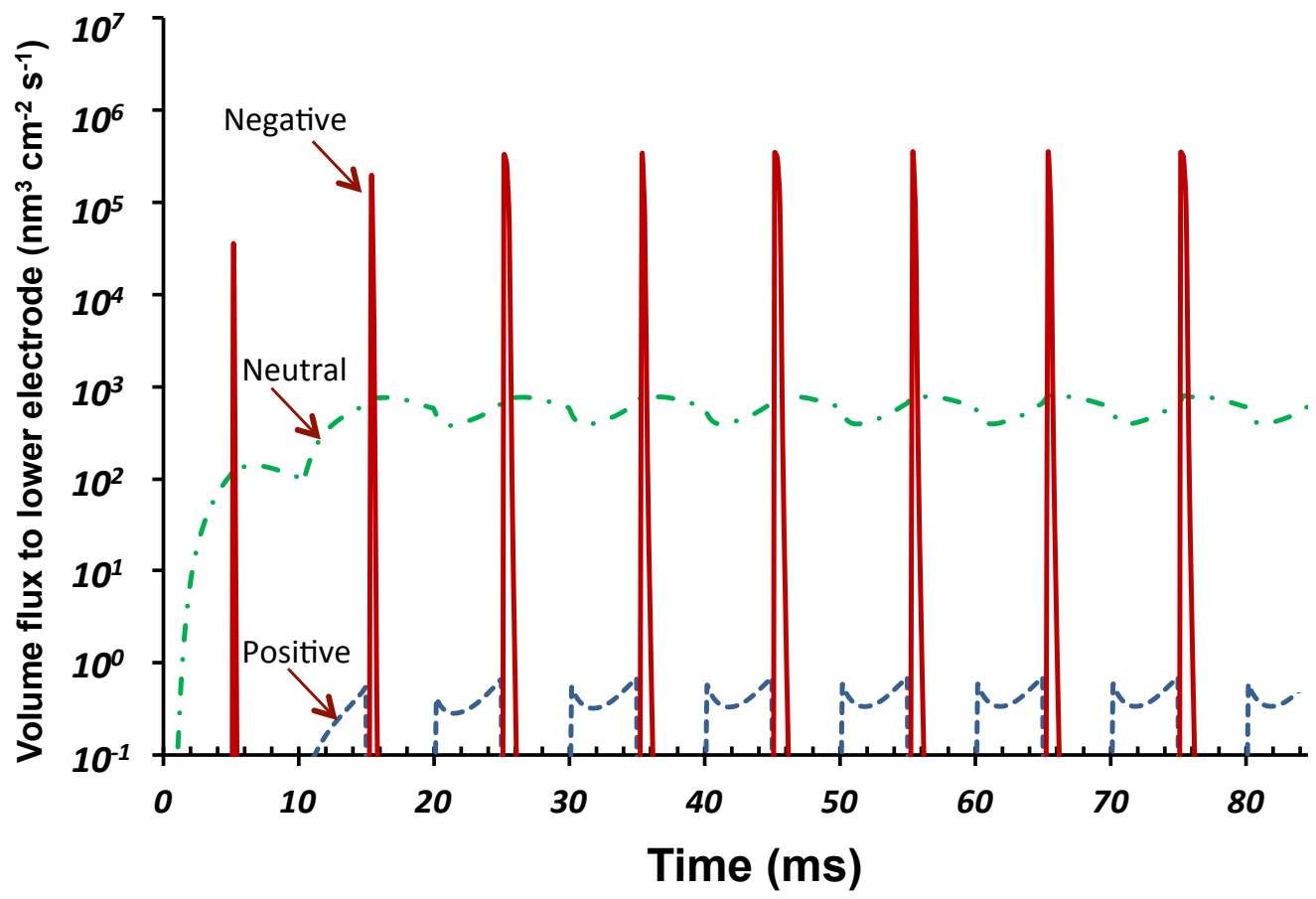

Figure 6. Volumetric fluxes of negative, neutral and positive nanoparticles to lower electrode for first eight RF pulses, base case conditions.

trode, while negative nanoparticles that form are trapped in the bulk plasma, and no flux of positive nanoparticles appears above the lower limit of the graph. At time $t=5 \mathrm{~ms}$ the RF power is turned off, and quickly one observes a sharp pulse of negative nanoparticles collected by the lower electrode. During the 5-ms afterglow the flux of neutral nanoparticles to the electrode continues but decays. Then at $t=10 \mathrm{~ms}$ the RF power is turned on again and the process repeats. The volume fluxes to the lower electrode of negative and neutral nanoparticles during the second pulse are both almost an order of magnitude higher than in the first pulse, and a flux of positive nanoparticles appears during the RF power-on phase. By the third pulse, commencing at $t=20$ $\mathrm{ms}$, the system is close to a periodic steady state, and by the fourth pulse onward, little difference is seen in the periodic behavior of the fluxes to the lower electrode of the separate populations of negative, neutral and positive nanoparticles.

It is thus evident that a periodic steady state is quickly achieved, and that the results shown in Figs. 2-5 for density profiles and size distributions at various times during a single pulse, which happens in this case to be the eighth pulse, are reproducible from pulse to pulse. In particular, following an initial transient period lasting two or three pulses during which the popula- 
tion of nanoparticles establishes itself, the conditions at the end of each afterglow phase (Fig. 5) are repeatable. At that time all of the negative nanoparticles have been collected, and only very small neutral nanoparticles remain. When the RF power is turned back on, some of these neutral nanoparticles charge negatively while new nanoparticles are created by fresh nucleation. The trapped, negatively charged nanoparticles grow, and the cycle repeats itself.

For base case conditions after a periodic steady state is achieved, the average diameter of nanoparticles that reach the lower electrode equals $1.26 \mathrm{~nm}$ for negative particles, $0.55 \mathrm{~nm}$ for neutral particles, and $0.61 \mathrm{~nm}$ for positive nanoparticles. Thus each negatively charged nanoparticle contributes more volume (mass) to film deposition than each neutral nanoparticle. Additionally, since volumetric flux equals the product of volumetric density times velocity, the negatively charged nanoparticles, accelerated by the positive DC bias applied to the electrode during the afterglow, contribute even more to the flux. Averaged over the entire cycle, for base case conditions, the volume flux of negative nanoparticles equals $\sim 96 \%$ of the total nanoparticle volume flux.

We find that a periodic steady state is achieved under some conditions but not others. The most important factor that determines whether a periodic state is reached is whether all negative nanoparticles are collected during the RF power-off phase of each pulse. In the base case the negative nanoparticles are all collected on a time scale that is considerably shorter than the length of the off phase. However if the off phase is sufficiently short, for example $1 \mathrm{~ms}$, all other parameters being held the same as in the base case, then not all negative nanoparticles are collected. In fact, because of the size classification noted above, it is the largest nanoparticles that are not collected. When the RF power is turned back on these relatively large nanoparticles are pulled back to the center of the plasma and resume their growth. Thus the uncollected nanoparticles in the bulk plasma become larger and larger from pulse to pulse. This behavior is illustrated in Fig. 7, which compares particle size distributions at the end of the RF-on phase in the 4th and 7 th cycles, for a case where the RF power-off time equals only $1 \mathrm{~ms}$ - too short a time for all of the nanoparticles to be collected-with all other conditions the same as in the base case. As the particles become larger they also become more and more negatively charged, finally perturbing the plasma by affecting quantities such as electron density and temperature. Under such conditions a periodic steady state cannot be established. 

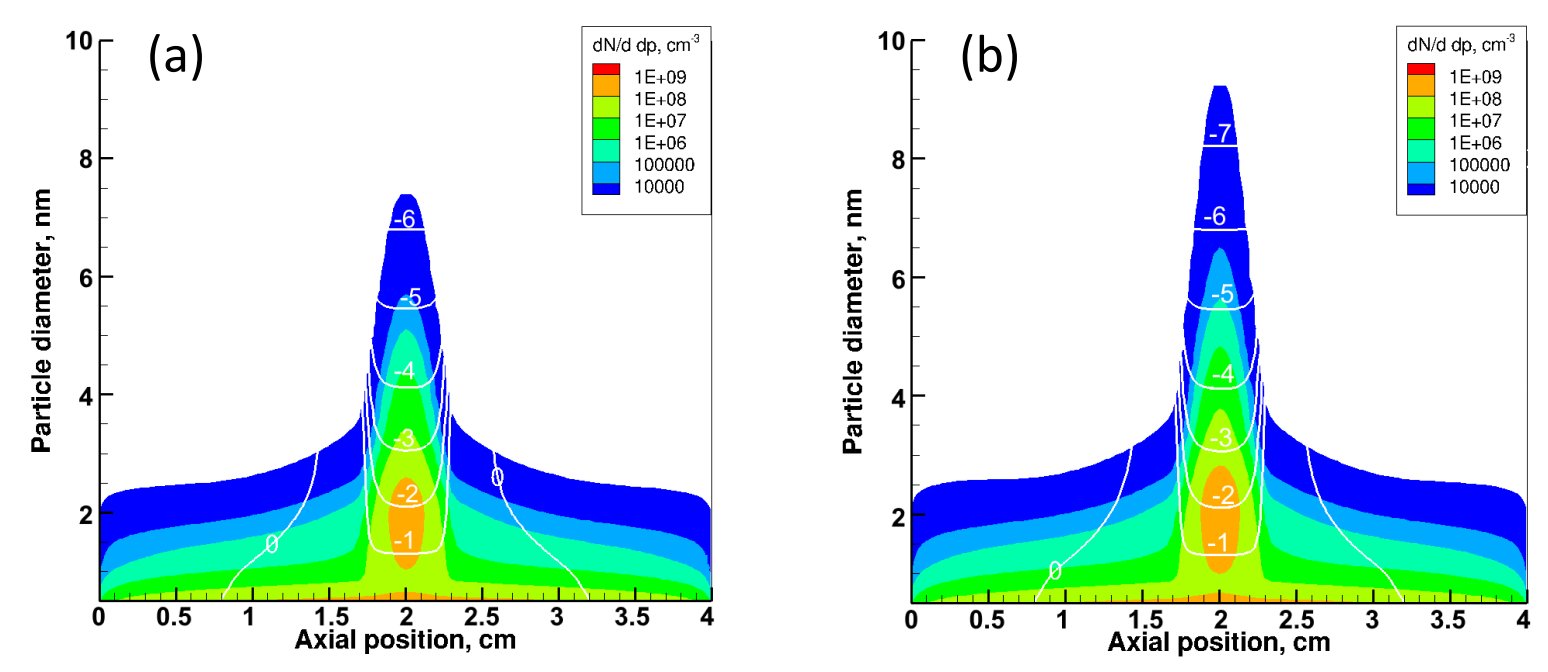

Figure 7. Profiles of particle size distribution (color contours) and average charge (white lines) at end of RF power-on phase of the 4th (a) and 7th (b) pulse cycles, for a case with RF power-off time equal to $1 \mathrm{~ms}$. All other conditions are same as in base case.

In addition to the duration of the RF off phase, other operating parameters can also affect whether or not a periodic state is achieved. The value of the DC bias applied during the off phase affects the time required to collect all the negative nanoparticles. Thus, for a given duration of the off phase, the DC bias must exceed some minimum value. Parameters such as pressure, RF voltage and the RF power-on time also determine whether a periodic steady state can be achieved, primarily because they affect the size to which the negative nanoparticles grow during the RF on time, and therefore the time required to collect them. Pressure also affects the time required to collect the negative nanoparticles, for given particle size, as it affects their mobility. Quantitive examples of these effects are presented in the next section.

\subsection{Effects of operating parameters}

In addition to achieving pulse-to-pulse reproducibility of the nanoparticle fluxes to a substrate, it is desirable to control characteristics of the nanoparticle fluxes in the context of applications. Properties of interest include the kinetic energy per atom of impacting nanoparticles, the film growth rate due to nanoparticle deposition, and the fraction of this growth rate that is due to negatively-charged nanoparticles, which are attracted by the applied DC bias, as opposed to neutral nanoparticles, which deposit by much lower-velocity diffusion. Results presented below are all obtained after the system has reached a periodic steady state. 


\subsubsection{Nanoparticle impact energy}

The kinetic energy per atom of nanoparticles impacting the substrate is expected to be an important parameter that governs whether low-temperature epitaxial film growth can be achieved without causing film damage. As discussed in Section 1, molecular dynamics simulations of nanoparticle impact for a variety of materials suggests that the desirable impact energy to achieve these objectives is of the order $\sim 1 \mathrm{eV}$ per atom, which is sufficient to cause nanoparticle melting and flattening but not so high as to cause film damage. Clearly experiments are required to refine this estimate for given material systems.

The pulsed RF plasma discussed herein has several independently controllable operating parameters. These include plasma parameters such as pressure and RF voltage, and pulse parameters such as RF power on time and off time (equivalently, pulse frequency and duty cycle), as well as the value of the DC bias voltage applied during the afterglow phase of each cycle.

Figure 8 shows the effect of these parameters on the distribution of impact energies per atom of all nanoparticles having diameters greater than or equal to $1.0 \mathrm{~nm}$. The reason that we plot impact energies on a per-atom (equivalently, per-unit-mass) basis is that this is the most pertinent quantity for nanoparticle heating and melting. The cutoff at $1.0 \mathrm{~nm}$ is arbitrary-sub-nanometer particles as well as atomic and molecular ions typically have higher impact energies per atom than nanoparticles of the same charge, but because of their smaller momentum they are less likely to cause substrate damage, compared to larger nanoparticles with the same impact energy per atom. Of course, atomic and molecular ions with energies of several $10 \mathrm{~s} \mathrm{of} \mathrm{eV} \mathrm{could} \mathrm{still} \mathrm{cause}$ damage, but we do not consider these as nanoparticles.

Increasing the RF voltage (equivalently, the power deposited in the plasma) increases the dissociation of silane and the densities of radicals that grow the nanoparticles. Thus the nanoparticles grow larger during the RF power-on time. The average diameter of the negative nanoparticles in the $160-\mathrm{V}$ case equals $1.90 \mathrm{~nm}$, compared to $1.26 \mathrm{~nm}$ in the base (100-V) case. Even neglecting the greater drag on larger particles as they are attracted to the substrate, these particles, having more atoms, therefore have lower impact energy per atom than in the base case, for given DC bias applied to the collecting substrate. Increasing the pressure has a similar effect. In these simulations the argon-to-silane ratio is fixed at 30:1, so increasing the total pressure increases the silane partial pressure, and correspondingly increases the densities of radical species and hence 


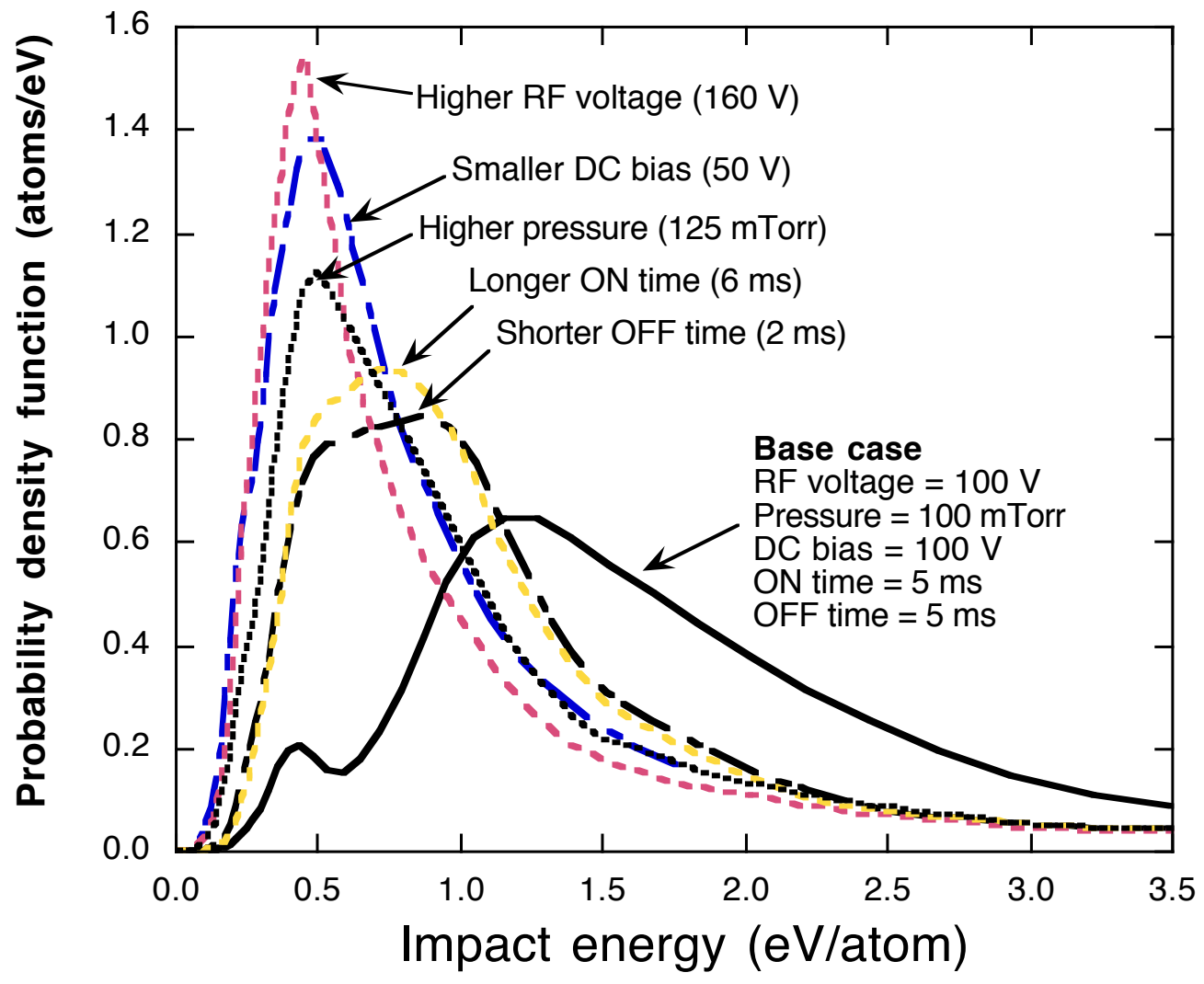

Figure 8. Distribution of impact energies per atom of negatively-charged nanoparticles greater than $1.0 \mathrm{~nm}$ in diameter, for the base case and for other cases where one parameter at a time is varied, all other parameters held the same as in the base case.

the sizes attained by nanoparticles during each RF power-on phase.

The DC bias voltage is the most straightforward control on particle impact energy, as it controls the (negative) potential drop experienced by the particles as they are transported to the substrate. For particles of given size, reducing the DC bias thus reduces the impact energy per atom. Increasing the RF power-on time allows the nanoparticles to grow larger; these larger particles impact with lower energy per atom. The effect of reducing the RF power-off time, provided it remains long enough to collect all of the negatively-charged nanoparticles, is that it also increases the average size of the impacting nanoparticles, but the reasons are more subtle than in the case of increasing the on time. When the off time is reduced, the neutral nanoparticles that remain uncollected at the end of the afterglow phase are on average larger. When the RF power is switched back on, many of these neutral nanoparticles attach an electron and are pulled back to the center. Hence the average size of the particles that are subsequently collected during the off 
phase is larger.

The fact that in all these cases one sees a distribution of impact energies per atom reflects several factors, including the existence of a distribution of nanoparticle sizes, and, for particles of given size, a distribution of the number of negative charges. For particles of given charge, the impact energy per atom decreases as particle size increases. This is due not only to the obvious fact that larger particles contain more atoms, but also results from the inverse relation between mass and mobility_larger particles experience more drag due to collisions as they approach the substrate, and thus reach a lower drift velocity. Furthermore, the electric field profile varies with time during the period when negatively-charged nanoparticles are collected by the lower electrode, because the electric potential profile is coupled by Poisson's equation to the changing density profiles of charged nanoparticles and ions. This is seen in Fig. 9, which shows the temporal evolution during the afterglow of the potential profile across the electrode gap, for base case conditions, for which the DC bias applied to the lower electrode equals $100 \mathrm{~V}$. The electric field

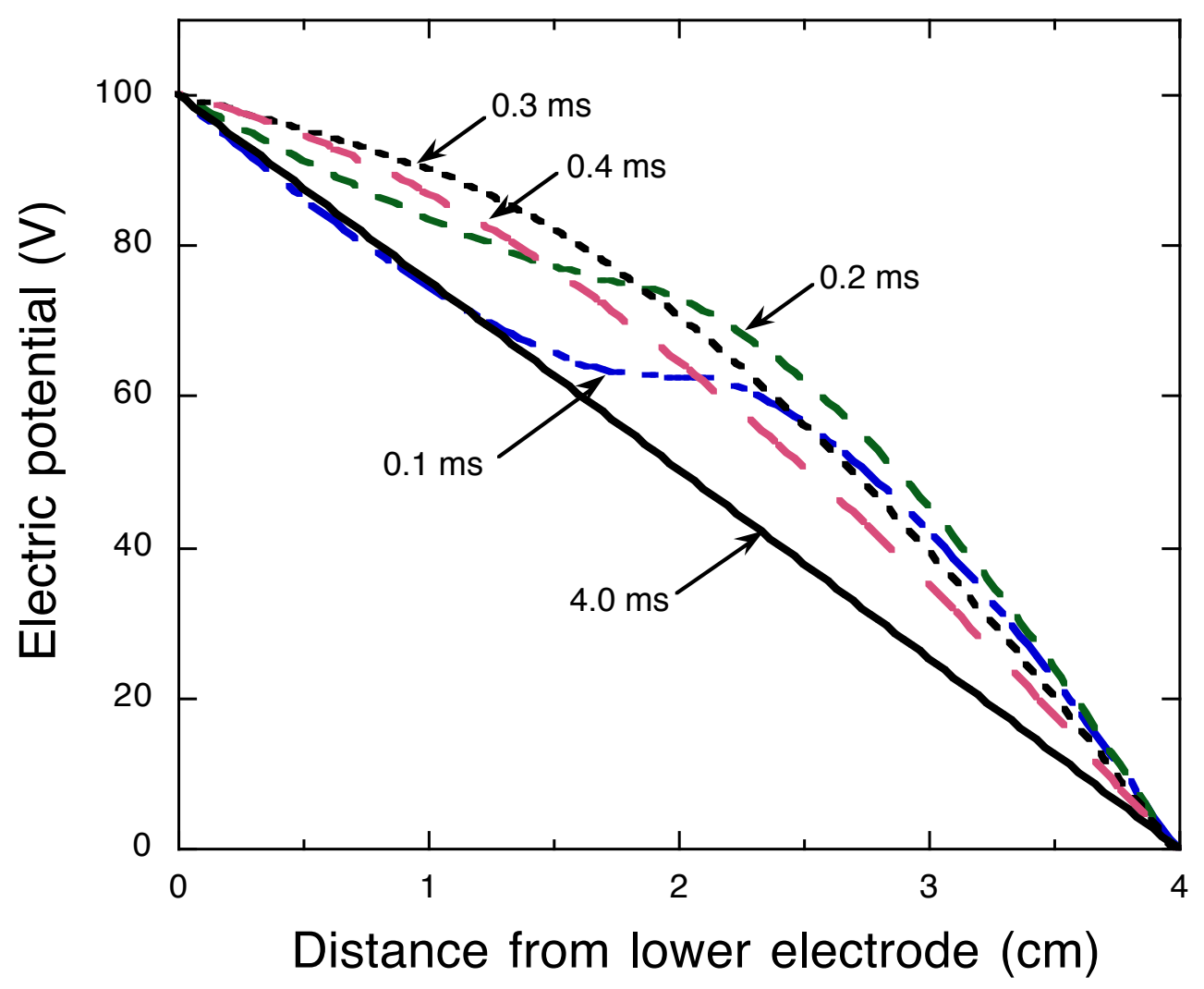

Figure 9. Profiles across electrode gap of electric potential at various times into afterglow phase of a pulse, for base case conditions, including 100-V DC bias applied to lower electrode. 
due to the externally applied DC bias is superimposed on the self-generated electric field that results from the changing charge-carrier profiles seen in Figs. 3(c), 4(c) and 5(c). And insofar as the magnitude of the electric field directly at or near the lower electrode determines the impact velocity, it is evident from Fig. 9 that the slope of the potential profile near the substrate, i.e. the field, varies with time. In turn this causes the nanoparticle impact velocity to vary with time, thereby broadening the impact energy distribution.

\subsubsection{Nanoparticle deposition rate}

The nanoparticle deposition rate is an important metric that can be expected to affect the commercial viability of the proposed scheme. In Fig. 10 we show the deposition rate calculated for the same six cases that are shown in Fig. 8, averaged in each case over multiple cycles after the system has reached periodic state. Clearly the deposition rate can be strongly affected by adjustable parameters. A small increase in pressure, from 100 mTorr to 125 mTorr, is seen to cause a more than 10-fold increase in deposition rate, as does an increase in RF voltage from 100

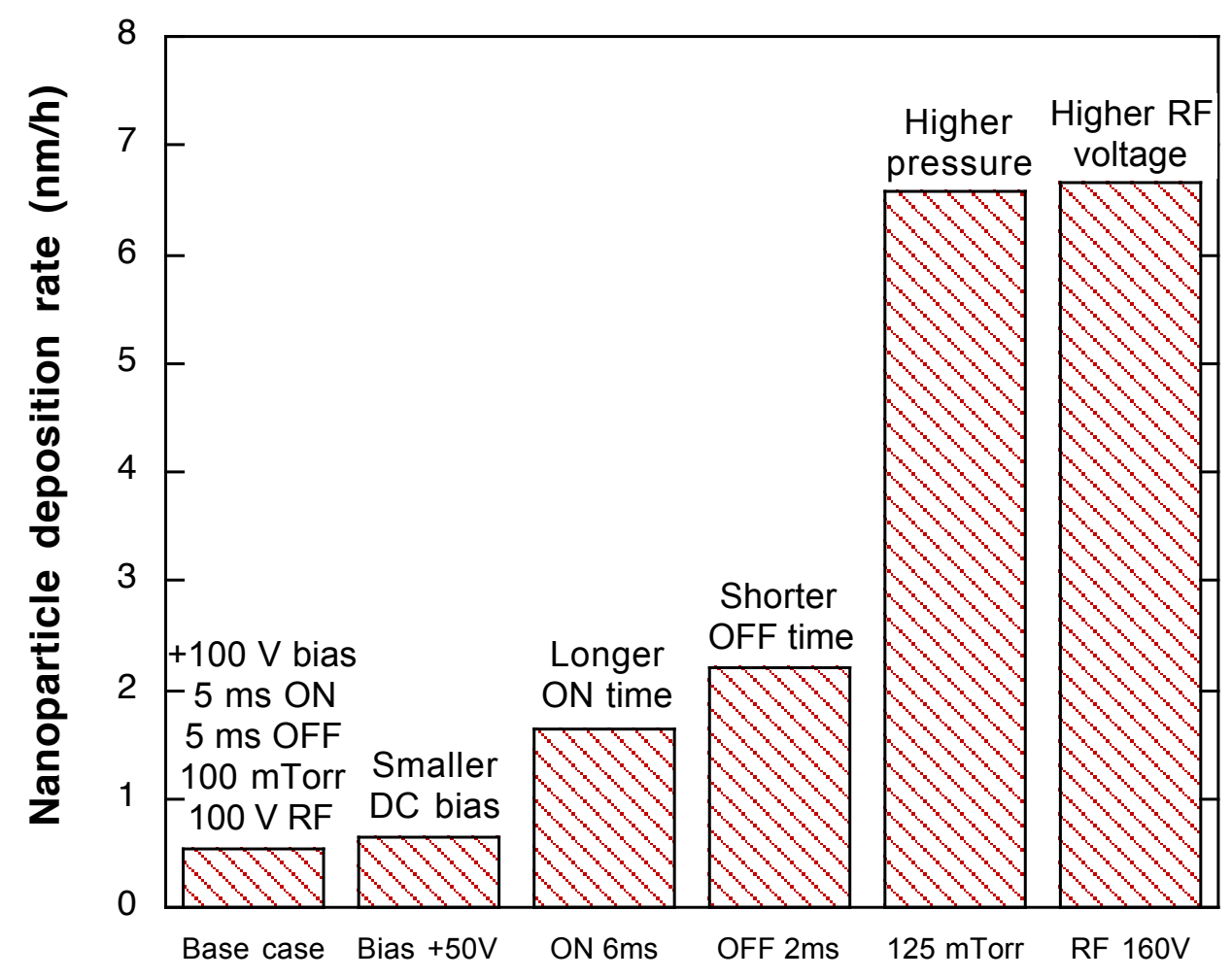

Figure 10. Nanoparticle deposition rate averaged over several cycles after system reaches periodic steady state, all six cases. 
to $160 \mathrm{~V}$. In both of those cases the higher deposition rate is a consequence of the greater conversion of silane to nanoparticles during the RF power-on phase of each pulse. Reducing the power-off time also significantly increases the deposition rate. This is partly because each pulse has less "wasted time" after all negative nanoparticles have been collected but before the next power-on phase begins, and partly because, as discussed in Sect. 3.3.1, the average size of the collected negative nanoparticles is larger. Longer power-on time also increases deposition rate: by increasing the duty cycle, a larger fraction of each cycle is used for nanoparticle growth. Changing the DC bias voltage that is applied during the off phase is seen to have only a slight effect on deposition rate. This is partly because in both cases shown the RF off time is long enough to collect all of the negative nanoparticles.

The nanoparticle deposition rates predicted in these simulations are low from the viewpoint of practical viability, where rates exceeding $\sim 100 \mathrm{~nm} / \mathrm{h}$ are likely required. Nevertheless, we are far from maximizing the deposition rate here, as it can also be affected by parameters that were fixed in these simulations, such as the silane flow rate. Furthermore, the conversion efficiency for the conditions assumed here-defined as the fraction of silicon atoms that enter the system in the form of silane that deposit in the form of nanoparticles - is low. For the cases of $\sim 7 \mathrm{~nm} / \mathrm{h}$ deposition rate seen in Fig. 10, the conversion efficiency equals $\sim 0.3 \%$. Thus there is considerable room for improvement in the deposition rate, as suggested by the strong sensitivity of the deposition rate to pressure and RF voltage (equivalently, power input), both of which increase the conversion efficiency. The main point is that the nanoparticle deposition rate is strongly affected by controllable process parameters.

It should also be noted that we have not modelled film growth on the substrate by chemical vapor deposition (CVD), which could in principle occur concurrently with nanoparticle deposition. One motivation of the proposed RF pulsing scheme is to deposit monocrystalline films at low substrate temperature, where CVD film growth might be avoided or at least minimized.

\subsubsection{Fraction of collected nanoparticles that are neutral}

As is evident from Figs. 2-6, a considerable fraction of nanoparticles produced in the plasma are non-negative, mostly neutral. As the neutral particles are not affected by the electric fields in either the RF power on or off phases of each cycle, they continually deposit on the collection substrate by Brownian diffusion. If the goal of the process is to produce epitaxial film 
growth by high-velocity impact of nanoparticles, these particles that deposit by "soft landing" represent potential defects that can disrupt the epitaxial growth. Therefore it is desirable to minimize the volume or mass fraction of the film that is comprised of these neutral nanoparticles.

Figure 11 shows the volume fraction of the total nanoparticle deposit on the collection substrate that consists of neutral nanoparticles, also for the same six cases as in Fig. 8. In each case the calculation is performed after the system has reached a periodic steady state. In Fig. 6 one observes that the peak in the flux of negative nanoparticles is $\sim 10^{3}$ times higher than for neutral nanoparticles. However the pulse of negative nanoparticles delivered to the substrate is brief, whereas deposition of neutral nanoparticles is continuous, occurring when the RF power is on as well as during the afterglow. Nevertheless, Fig. 11 indicates that the neutral contribution to the volume fraction of the nanoparticle film can be made quite small. Among the cases tested, the smallest value found was $0.7 \%$, for the case of $160-\mathrm{V}$ RF voltage. It is not unreasonable to expect that by further parameter optimization the contribution to the film of neutral nanoparticles can be made smaller than $0.1 \%$. Since neutral nanoparticles that deposit on the substrate are

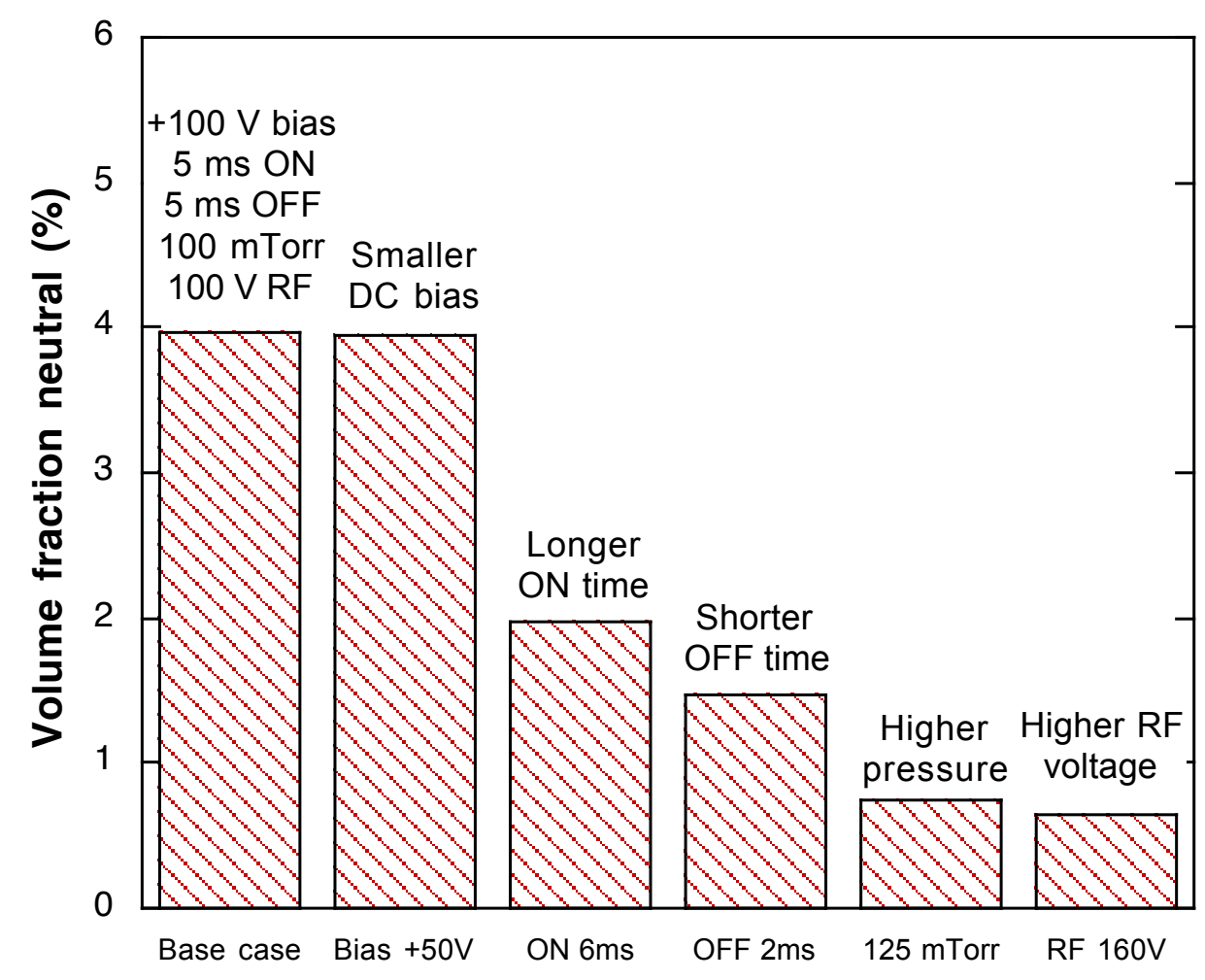

Figure 11. Volume fraction of deposited nanoparticles that is comprised of neutral particles, all six cases. 
quite small (e.g., with an average diameter of $0.55 \mathrm{~nm}$ in the base case), it seems plausible that such a small contribution of low-velocity neutrals would not prevent the epitaxial film growth fostered by high-velocity impact of negative nanoparticles.

The effects of the different parameters on this metric are straightforward to understand. Longer RF power on times promote growth of larger nanoparticles, and the larger particles are effectively all negatively charged, hence the neutral fraction is smaller. Shorter RF power off times reduce the "wasted" part of each pulse cycle during which particles are not growing and only neutral nanoparticles are depositing. Increasing the pressure, in addition to growing larger negatively-charged particles, reduces the diffusion loss rate of neutral particles. Increasing the RF voltage, in addition to increasing particle size, increases charging, and thus reduces the fraction of particles that are neutral.

\section{Conclusions}

In this work we have modeled a pulsed RF argon-silane plasma to explore the feasibility of delivering controlled fluxes of silicon nanoparticles to a substrate to promote epitaxial silicon film growth. The pulsing scheme involves application of a positive DC bias to the particle collection substrate during the afterglow phase of each pulse. Our results indicate that with this approach it should be possible to rapidly achieve a periodic steady state in which the properties of the nanoparticle fluxes to the substrate are reproducible from pulse-to-pulse. In the regime where this condition is satisfied, several adjustable operating parameters exist that may allow one to achieve several practical objectives. All of the negatively charged nanoparticles produced during each pulse are collected. Average diameters of the collected nanoparticles can be kept below 2 or $3 \mathrm{~nm}$. This method does not produce monoenergetic beams of nanoparticles, for several reasons: the impacting nanoparticles are inherently polydisperse; there will also be a distribution with respect to particle charge; and the electric field profile during the afterglow itself changes with time, because it is coupled to the changing density profiles of charge carriers. Nevertheless, nanoparticle impact energies on the collection substrate can be maintained in the $\sim 1 \mathrm{eV} /$ atom range thought to be conducive to nanoparticle melting and epitaxial growth without causing damage to the underlying film, and the distribution of impact energies can be made reasonably narrow. Additionally, the volume fraction of the deposited nanoparticles that are neutral and that deposit by low-velocity Brownian diffusion can be made less than $1 \%$, and perhaps less 
than $0.1 \%$, and these neutral nanoparticles are significantly smaller than the negatively-charged nanoparticles that deposit by high-velocity impact.

The predicted rates of film growth by nanoparticle impact in these simulations are perhaps too low by more than an order of magnitude with respect to the values required for a commercially viable process. However, we did not attempt to vary conditions so as to optimize deposition rate. Furthermore, it should be noted that we have here explored plasmas consisting of silane highly diluted in argon, at pressures around 100 mTorr, whereas the experiments discussed in Ref. 1 involved a rather different set of conditions: hydrogen-silane plasmas with lower dilution ratios, and at pressures greater than 1 Torr. In Ref. 1, these conditions produced epitaxial films with a growth rate of $600 \mathrm{~nm} / \mathrm{h}$. Simulations of these higher pressure hydrogen-silane plasmas are currently in progress, and will be reported in a subsequent publication.

\section{Acknowledgments}

This work was partially supported by the U.S. National Science Foundation (CHE124752) and the U.S. Dept. of Energy Office of Fusion Energy Science (DE-SC0001939).

\section{References}

1. Roca i Cabarrocas P, Cariou R, Labrune M (2012) Low temperature plasma deposition of silicon thin films: From amorphous to crystalline. J. Non-Cryst. Sol. 358:2000-2003

2. Bouchoule A, Boufendi L (1993) Particulate formation and dusty plasma behaviour in argonsilane RF discharge. Plasma Sources Sci. Technol. 2:204-213

3. Roth RM, Spears KG, Stein GD, Wong G (1985) Spatial dependence of particle light scattering in an rf silane discharge. Appl. Phys. Lett. 46:253-255

4. Watanabe Y, Shiratani M, Yamashita M (1992) Observation of growing kinetics of particles in a helium-diluted silane rf plasma Appl. Phys. Lett. 61:1510-1512

5. Mangolini L, Thimsen E, Kortshagen U (2005) High-yield plasma synthesis of luminescent silicon nanocrystals. Nano Lett. 5:655-659

6. Howling AA, Dorier J-L, Hollenstein C (1993) Negative ion mass spectra and particulate formation in radio frequency silane plasma deposition experiments. Appl. Phys. Lett. 62:1341-1343

7. Hwang NM, Lee, DK (2010) Charged nanoparticles in thin film and nanostructure growth by chemical vapour deposition. J. Phys. D 43:483001

8. Chaabane N, Suendo V, Vach H, Roca i Cabarrocas P (2006) Soft landing of silicon nanocrystals in plasma enhanced chemical vapor deposition Appl. Phys. Lett. 88:2031111

9. Haberland H, Insepov Z, Moseler M (1995) Molecular-dynamics simulation of thin-film growth by energetic cluster impact. Phys. Rev. B 51:11061-11067

10. Cheng H-P, Landman U (1994) Controlled deposition and classification of copper nanoclusters. J. Phys. Chem. 98:3527-3537 
11. Suri M, Dumitrică T (2008) Efficient sticking of surface-passivated Si nanospheres via phase-transition plasticity. Phys. Rev. B 78:081405

12. Warthesen SJ, Girshick SL (2007) Numerical simulation of the spatiotemporal evolution of a nanoparticle-plasma system. Plasma Chem. Plasma Process. 27:292-310

13. Agarwal P, Girshick SL (2012) Sectional modeling of nanoparticle size and charge distributions in dusty plasmas. Plasma Sources Sci. Technol. 21:055023

14. Agarwal P, Girshick SL (2014) Numerical modeling of the spatiotemporal behavior of an RF argon-silane plasma with dust particle nucleation and growth. Plasma Chem. Plasma Process. 34:489-503

15. Allen JE (1992) Probe theory-the orbital motion approach. Phys. Scripta 45:497-503

16. Gallagher A (2000) A model of particle growth in silane discharges. Phys. Rev. E 62:26902706

17. Le Picard R, Girshick SL (2016) The effect of single-particle charge limits on charge distributions in dusty plasmas. J. Phys. D 49:095201

18. Ravi L, Girshick SL (2009) Coagulation of nanoparticles in a plasma. Phys. Rev. E 79:026408-9

19. Huang DD, Seinfeld JH, Okuyama K (1991) Image potential between a charged particle and an uncharged particle in aerosol coagulation-Enhancement in all size regimes and interplay with van der Waals forces. J. Coll. Interface Sci. 141:191-198

20. Agarwal P (2012) Numerical modeling of plasmas in which nanoparticles nucleate and grow. $\mathrm{Ph} . \mathrm{D}$. thesis, U. Minnesota, Minneapolis

21. Ramamurthi B, Economou DJ (2002) Pulsed-power plasma reactors: two-dimensional electropositive discharge simulation in a GEC reference cell. Plasma Sources Sci. Technol. 11:324-332.

22. Hebner GA, Fleddermann CB (1997) Characterization of pulse-modulated inductively coupled plasmas in argon and chlorine. J. Appl. Phys. 82:2814-2821 